\title{
ROLE OF INTEGRATED POSITRON EMISSION TOMOGRAPHY/COMPUTED TOMOGRAPHY IN DETECTING RECURRENT OVARIAN CANCER IN PATIENTS WITH RISING CA-125 LEVELS
}

\author{
Fozia Naz ${ }^{1}$, Ahmad Murtaza ${ }^{2}$, Khurram Aftab ${ }^{3}$, Zia S. Faruqui ${ }^{1}$, Humayun Bashir ${ }^{4}$ \\ ${ }^{1}$ Department of Radiology, Shaukat Khanum Memorial Cancer Hospital and Research Centre, Lahore, Pakistan, \\ ${ }^{2}$ Department of Radiology, King Saud University Hospital, Riyadh, Saudi Arabia, ${ }^{3}$ Department of Radiology, Sheikh \\ Khalifa Medical City, Abu Dhabi, UAE, ${ }^{4}$ Department of Nuclear Medicine, Shaukat Khanum Memorial Cancer \\ Hospital and Research Centre, Lahore. Pakistan
}

Received: 1 January 2015 / Accepted: 11 September 2015

\begin{abstract}
Purpose: The purpose of this study was to assess the sensitivity and diagnostic accuracy of integrated positron emission tomography/computed tomography (PET/CT) in detecting recurrent ovarian cancer in treated patients presenting with rising CA-125 levels during clinical follow-up and compare it with those of CT alone.

Materials and Methods: This was a retrospective study. We evaluated 45 patients with pathologically proven ovarian carcinoma who underwent PET/CT during October 2010-November 2013 at our institution for suspected relapse; IRB deemed that approval for this retrospective study was not required. Of these, 35 patients who presented with rising CA-125 levels during clinical follow-up were included in this study. Remaining 10 patients were excluded as they had normal CA-125 levels. At least three previous consecutive CA-125 readings and initial conventional imaging before fluorodeoxyglucose-PET/CT were noted. Sensitivity and diagnostic accuracy for tumour detection with PET/CT and CT alone were calculated; histological analysis after biopsy/second-look surgery or clinicalradiologic follow-up/response to chemotherapy was taken as reference standard. K statistics (Cohen K) was used for statistical analysis.
\end{abstract}

Results: Of 35 patients with suspected relapse, one patient was lost to follow up. 30 patients were documented to have relapsed, while in four patients, recurrence was not identified either on CT or PET-CT and they were proved to be disease free on 2-year follow-up. Amongst these relapsed patients, 10/30 cases were proven histologically, 3 with imaging-guided biopsy and 7 with second-look surgery, whereas 20/30 were confirmed on clinical/radiological follow-up (ranging from 3 to 6 months) or by response to chemotherapy on subsequent imaging. Of 30 patients with relapse, PET-CT highlighted recurrence in 27. Sensitivity, specificity, positive predictive value, negative predictive value, and diagnostic accuracy of integrated PET/CT were calculated to be $90 \%, 75 \%, 96 \%, 50 \%$, and $88 \%$, respectively. CT alone detected recurrence in 20 patients. Sensitivity, specificity, positive predictive value, negative predictive value, and diagnostic accuracy of CT were calculated to be $73.3 \%, 100 \%, 100 \%, 33 \%$, and $76 \%$, respectively.

Conclusion: PET/CT is a highly sensitive and accurate post-therapy surveillance modality for the detection of recurrent ovarian cancer in patients with rising tumour markers as compared to CT alone.

Key words: CA125, fluorodeoxyglucose, ovarian cancer, positron emission tomography/computed tomography, recurrence

Correspondence: Dr. Fozia Naz, Department of Radiology,

Shaukat Khanum Memorial Cancer

Hospital and Research Centre, Lahore, Pakistan.

Email: n_fozia@yahoo.com

\section{Introduction}

Ovarian cancer is the most common gynaecological malignancy ${ }^{[1]}$ Treatment of choice is cytoreductive surgery 
with post-operative chemotherapy such as paclitaxel and taxane preparations ${ }^{[2,3]}$ Despite adequate treatment and complete response, recurrence is common. Estimated recurrence rate is $60 \%$ in patients with advanced stage at the time of diagnosis. Patients usually develop abdominal relapse. ${ }^{[4]}$ Clinical follow-up of ovarian cancer patients is done with serial measurements of the serum CA-125 levels. In patients with elevated CA- 125 levels at baseline that normalise post-treatment, progressively increasing CA-125 levels are indicative of recurrent ovarian cancer, even in the absence of clinical or radiologic findings that warrant detailed evaluation. ${ }^{[5,6]}$ Multiple imaging modalities are used to detect cancer recurrence. It includes ultrasonography, computed tomography (CT) scan, magnetic resonance imaging (MRI), and positron emission tomography (PET). Small-volume disease $<2 \mathrm{~cm}$ can be missed on anatomic imaging alone due to the visceral metastasis. Functional imaging that uses uptake of radioisotope labelled glucose by tumour helps to identify the recurrent disease as it increases contrast between tumour and normal structures. ${ }^{[7,8]}$ Metastases from ovarian cancer are primarily peritoneal rather than parenchymal sites. Small-volume metastatic deposits on the visceral surfaces are diagnostic challenge if interpreted with anatomic imaging alone such as CT and MRI. ${ }^{[9,10]}$ Integrated PET/CT is superior for the detection of ovarian cancer relapse than anatomic imaging such as CT and MRI, with the use of a metabolic tracer and simultaneous acquisition of anatomic data to determine the exact location of lesions. ${ }^{[11]}$ Moreover, compared to anatomic imaging, PET/CT is used to survey the entire body to detect recurrence in multiple sites that are crucial for further treatment planning to avoid further relapse. The purpose of the study is to evaluate and compare the diagnostic accuracy of integrated PET-CT to CT alone in patients suspected for recurrent ovarian carcinoma based on elevated tumour markers.

\section{Materials and Methods}

\section{Patients}

The retrospective study evaluated 45 patients of ovarian carcinoma who underwent PET/CT during October 2010November 2013. Of these, 35 patients who presented with rising CA-125 levels during clinical follow-up were included in this study. Remaining 10 patients were excluded as they had normal CA-125 in spite of the histopathologically proven ovarian carcinoma.
This was a retrospective study collecting data from archives/picture archiving and communication system (PACS), Department of Radiology. The protocol to acquire PET/CT in SKMCH is $>4$-h fast before the examination. Patients are asked to avoid high protein diet $24 \mathrm{~h}$ before scan. After recording demographic data, history, physical examination, informed consent, and blood sugar level test, the patient rests for a minimum of $15 \mathrm{~min}$, to reduce any muscular activity, which might interfere with tracer uptake. An intravenous injection of $18 \mathrm{~F}$-fluorodeoxyglucose (FDG) is then administered. After $45 \mathrm{~min}$, the patient is positioned in supine for PET-CT imaging. X-ray tomogram is acquired followed by whole-body CT imaging with contrast and then PET imaging thereafter. PET-CT software reconstruction was done, and only CT, only PET, and fused PET-CT images were displayed. A whole-body scan, which usually starts from mid-thigh to the top of the head, is acquired. FDG imaging protocol acquires slices with a thickness of 2-3 mm. All images are interpreted on a PACS workstation. Curved and multiplanar reformations are obtained at a dedicated post-processing workstation.

\section{Image analysis}

The images were visually assessed by a board-certified radiologist and nuclear medicine physician with PET expertise independently. Both FDG-PET and the CT portion of the PET/CT are interpreted in independent sessions by radiologist and nuclear physician, and they were not aware of each other's findings. An increase in FDG uptake was compared with the corresponding anatomical finding on CT scan images. An abnormal CT finding with increase in FDG uptake was interpreted as positive for recurrent disease. Any structural abnormality found on CT but without FDG uptake on PET imaging was interpreted as negative findings.

\section{Results}

Of 35 patients with suspected relapse on the basis of raised CA-125 levels, one patient was lost to follow up. 30 patients were documented to have relapse on imaging, while four patients were proved to be disease free on 2-year follow-up. Of 30 patients with relapse PET-CT highlighted possible recurrence in 28, 1 patient was false positive as detected hypermetabolic nodes were due to inflammation. PET-CT missed recurrence in three patients due to small size of 
lesion who subsequently presented with disease relapse large enough to be identifiable on anatomical imaging alone. 10/30 cases were histologically proven with imaging-guided biopsy and 7 with second-look surgery, whereas 20/30 were confirmed on clinical/radiological follow-up (ranging from 3 to 6 months) or by response to chemotherapy on subsequent imaging. Sensitivity, specificity, positive predictive value, negative predictive value, and diagnostic accuracy of integrated PET/CT were calculated to be $90 \%$, $75 \%, 96 \%, 50 \%$, and $88 \%$, respectively.

CT alone correctly detected recurrence in 22 patients. Sensitivity, specificity, positive predictive value, negative predictive value, and diagnostic accuracy of CT were calculated to be $73.3 \%, 100 \%, 100 \%, 33 \%$, and $76 \%$, respectively [Table 1 and Graph 1]. There were 8 falsenegative patients. $5 / 8$ false-negative patients were positive on PET-CT, while 3/8 were negative on both CT and PET$\mathrm{CT}$ and subsequently presented with relapse on continued follow-up.

\section{Discussion}

According to the National Cancer Institute consensus statement for ovarian cancer, the follow-up for treated

\section{Table 1: CT versus PET-CT}

\begin{tabular}{l|c|c}
\hline Evaluation & CT (\%) & PET-CT (\%) \\
\hline Sensitivity & 73.30 & 90 \\
\hline Specificity & 100 & 75 \\
\hline Diagnostic accuracy & 76 & 88 \\
\hline Positive predictive value & 100 & 96 \\
\hline Negative predictive value & 33 & 50 \\
\hline
\end{tabular}

PET-CT: Positron emission tomography-computed tomography

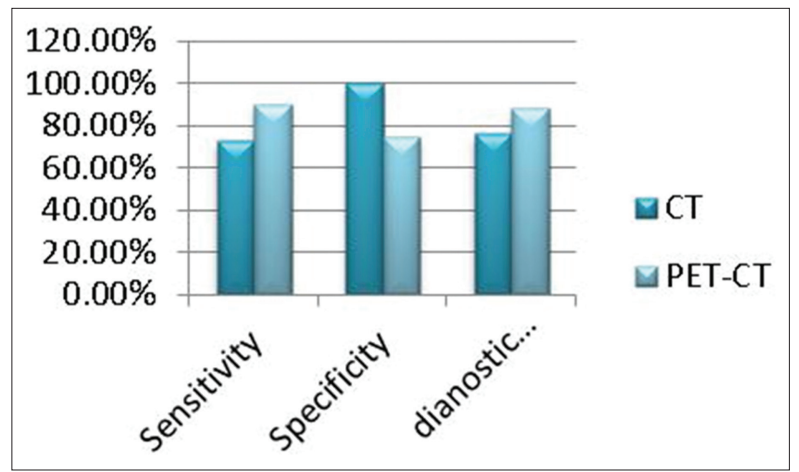

Graph 1: Comparison between CT and PET-CT patients of ovarian cancer should include routine clinical history, physical examination, ultrasound, and tri-monthly serum CA 125 determination for the first 2 years and then less frequently afterwards. ${ }^{[12]}$ However, one study showed that, in surveillance programmes, its pooled specificity was relatively low $0.69 .{ }^{[13]}$ Therefore, non-recurrent conditions such as infections may produce elevated levels. Similar thing noted in four of our patients as CA-125 levels was elevated while imaging did not detect any recurrence and the patient remained asymptomatic on 2-year follow-up. Raised CA-125 levels in those patients were due to TB that was proved histologically.

Most of the clinicians perform surveillance in treated ovarian cancer patients with biochemical markers such as CA-125 levels and imaging. Ultrasound, CT, MRI, and PET-CT all have been used for this purpose. Transvaginal ultrasound is superior to transabdominal scan to detect recurrence. Most of the studies concluded that diagnostic transabdominal ultrasound examination is unreliable modality for the detection of ovarian cancer recurrence particularly for the deposits located in the abdominal and pelvic peritoneum. ${ }^{[14]}$ The sensitivity of ultrasonography ranges from $74 \%$ to $96 \%$; its specificity ranges from $23 \%$ to $80 \%$. $^{[15]}$

CT scan uses morphological features to detect local pelvic recurrence and distant visceral/peritoneal metastasis. However, it is difficult to detect smaller deposits particularly those located on the visceral surface of bowel and micrometastases in radiologically benign appearing lymph nodes. It is also quite difficult to separately identify lesion from adjacent viscera as anatomy is usually distorted postoperatively. Spiral CT usually has high sensitivity of $85 \%-93 \%$ for the detection of larger peritoneal deposits. However, its sensitivity is low ranging from $25 \%-50 \%$ for sub-cm deposits. ${ }^{[9,10]}$

PET detects tumour recurrence due to increased radiotracer uptake by tumour cells on account of increased metabolic activity. However, anatomical details are lacking. Combined PET-CT improves diagnostic accuracy as it combines both features and thus becomes the most effective modality for the detection of recurrence and further treatment planning accordingly. PET/CT (sensitivity, 91\%, and specificity, 88\%) performed better than CT (sensitivity, 79\%, and specificity, 84\%) or MRI (sensitivity, 75\%, and specificity, 78\%). ${ }^{[16]}$ 


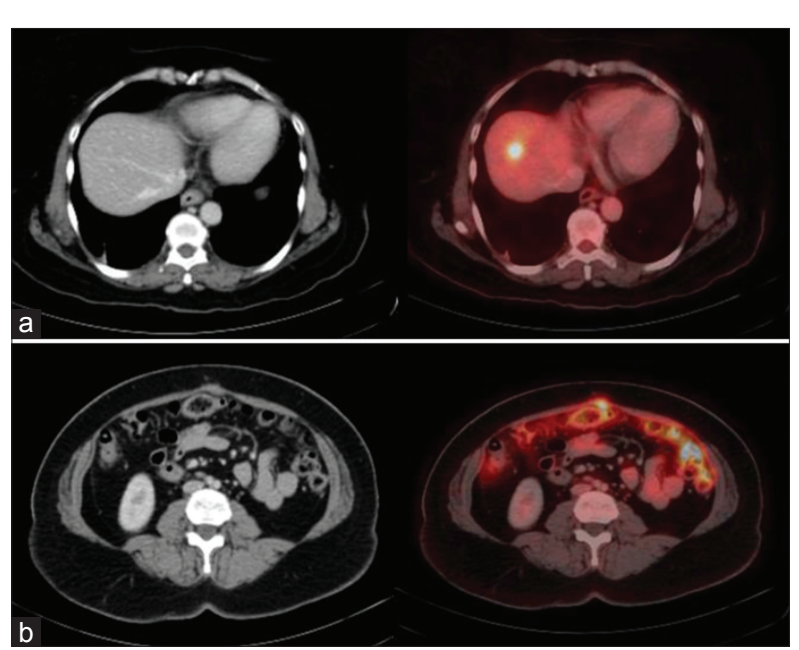

Figure 1: (a) Relapse identified as metabolically active hepatic metastasis on positron emission tomography/computed tomography (CT) imaging. No corresponding anatomical lesion identified on CT image. (b) A 35-year-old diagnosed patient of CA ovary with suspected relapse. Relapse documented as hypermetabolic soft tissue lesion in abdominal scar, further confirmed on FNAC

According to our study, sensitivity of PET-CT and CT is $90 \%$ and $66.7 \%$, respectively. PET-CT missed recurrence in 3 cases due to small size of the lesion who subsequently presented with disease relapse large enough to be identifiable on anatomical imaging alone.

In all the patients with recurrence detected on $\mathrm{CT}$ and PET-CT both, PET-CT still helped in treatment planning either by identifying other sites of disease or confidently excluding multiple tumour implants. It includes patients in whom there was solitary site of recurrence on CT while PET-CT detected recurrence at multiple sites who were offered chemotherapy rather than surgery and the patients who underwent surgery due to limited disease on PET-CT.

Our study has shown that PET/CT is a sensitive tool to assist in the early identification and recurrent ovarian cancer, amenable to secondary cytoreduction [Figure $1 \mathrm{a}$ and $\mathrm{b}$ ].

The precise PET-CT localisation of suspicious FDG uptake could lead to improved assessment of the extent of recurrent disease, thus allowing for optimised treatment plan for these patients.

\section{Conclusion}

FDG PET/CT is a highly sensitive and accurate for posttherapy surveillance in patients suspected of recurrent ovarian cancer with rising tumour markers as compared to $\mathrm{CT}$ alone.

\section{Conflict of Interest}

The authors declare that they have no conflict of interest.

\section{References}

1. Jemal A, Siegel R, Ward E, et al. Cancer statistics, 2009. CA Cancer J Clin 2009;59:225-49.

2. Stewart L, Advanced Ovarian Cancer Trialists Group. Chemotherapy for advanced ovarian cancer. Advanced ovarian cancer trialists group. Cochrane Database Syst Rev 2000;2:CD001418.

3. Aabo K, Adams M, Adnitt P, et al. Chemotherapy in advanced ovarian cancer: Four systematic meta-analyses of individual patient data from 37 randomized trials. Advanced ovarian cancer trialists' group. Br J Cancer 1998;78: 1479-87.

4. García-Velloso MJ, Jurado M, Ceamanos C, et al. Diagnostic accuracy of FDG PET in the follow-up of platinum-sensitive epithelial ovarian carcinoma. Eur J Nucl Med Mol Imaging 2007;34:1396-405.

5. Högberg T, Kågedal B. Long-term follow-up of ovarian cancer with monthly determinations of serum CA 125. Gynecol Oncol 1992;46:191-8.

6. Santillan A, Garg R, Zahurak ML, et al. Risk of epithelial ovarian cancer recurrence in patients with rising serum CA-125 levels within the normal range. J Clin Oncol 2005; 23:9338-43.

7. Buy JN, Moss AA, Ghossain MA, et al. Peritoneal implants from ovarian tumors: CT findings. Radiology 1988; 169:691-4.

8. Coakley FV, Choi PH, Gougoutas CA, et al. Peritoneal metastases: Detection with spiral CT in patients with ovarian cancer. Radiology 2002;223:495-9.

9. Kim HJ, Kim JK, Cho KS. CT features of serous surface papillary carcinoma of the ovary. AJR Am J Roentgenol 2004; 183:1721-4.

10. Qayyum A, Coakley FV, Westphalen AC, et al. Role of CT and MR imaging in predicting optimal cytoreduction of newly diagnosed primary epithelial ovarian cancer. Gynecol Oncol 2005;96:301-6.

11. Pannu HK, Bristow RE, Cohade C, et al. PET-CT in recurrent ovarian cancer: Initial observations. Radiographics 2004; 24:209-23.

12. National Institutes of Health. Ovarian cancer: Screening, treatment and follow-up. NIH Consens Statement 1994;12:1-30. 
13. Meyer T, Rustin GJ. Role of tumour markers in monitoring epithelial ovarian cancer. Br J Cancer 2000;82:1535-8.

14. Sugiyama T, Nishida T, Komai K, et al. Comparison of CA 125 assays with abdominopelvic computed tomography and transvaginal ultrasound in monitoring of ovarian cancer. Int J Gynaecol Obstet 1996;54:251-6.

15. Ferrazzi E, Zanetta G, Dordoni D, et al. Transvaginal ultrasonographic characterization of ovarian masses: Comparison of five scoring systems in a multicenter study. Ultrasound Obstet Gynecol 1997;10:192-7.

16. Gu P, Pan LL, Wu SQ, et al. CA 125, PET alone, PET-CT, $\mathrm{CT}$ and MRI in diagnosing recurrent ovarian carcinoma: A systematic review and meta-analysis. Eur J Radiol 2009; 71:164-74. 